\title{
2015 Recommendations for the management of polymyalgia rheumatica: a European League Against Rheumatism/American College of Rheumatology collaborative initiative
}

\author{
Christian Dejaco, ${ }^{1,2}$ Yogesh P Singh, ${ }^{2}$ Pablo Perel, ${ }^{3}$ Andrew Hutchings, ${ }^{4}$ \\ Dario Camellino, ${ }^{5}$ Sarah Mackie, ${ }^{6}$ Andy Abril, ${ }^{7}$ Artur Bachta, ${ }_{1}^{8}$ Peter Balint, ${ }^{9}$ \\ Kevin Barraclough, ${ }^{10}$ Lina Bianconi, ${ }_{11}^{11}$ Frank Buttgereit, ${ }^{12}$ Steven Carsons, ${ }^{13}$ \\ Daniel Ching, ${ }^{14}$ Maria Cid, ${ }^{15}$ Marco Cimmino, ${ }^{5}$ Andreas Diamantopoulos, ${ }^{16}$ \\ William Docken, ${ }^{17}$ Christina Duftner, ${ }_{1}^{18}$ Billy Fashanu, ${ }^{2}$ Kate Gilbert, ${ }^{19}$ \\ Pamela Hildreth, ${ }^{19}$ Jane Hollywood, ${ }^{2}$ David Jayne, ${ }^{20}$ Manuella Lima, ${ }^{21}$ \\ Ajesh Maharaj, ${ }^{22}$ Christian Mallen, ${ }^{23}$ Victor Martinez-Taboada ${ }^{24}$ Mehrdad Maz, ${ }_{1}{ }^{25}$ \\ Steven Merry, ${ }^{26}$ Jean Miller, ${ }^{19}$ Shunsuke Mori, ${ }^{27}$ Lorna Neill, ${ }^{19}$ Elisabeth Nordborg, ${ }^{28}$ \\ Jennifer Nott, ${ }^{19}$ Hannah Padbury, ${ }_{1}^{19}$ Colin Pease, ${ }^{6}$ Carlo Salvarani, ${ }^{29}$ \\ Michael Schirmer, ${ }^{18}$ Wolfgang Schmidt, ${ }^{30}$ Robert Spiera, ${ }^{31}$ David Tronnier, ${ }^{32}$ \\ Alexandre Wagner, ${ }^{33}$ Madeline Whitlock, ${ }^{2}$ Eric L Matteson, ${ }^{34}$ Bhaskar Dasgupta $^{2}$
}

Handling editor Tore K Kvien

- Additional material is published online only. To view please visit the journal online (http://dx.doi.org/10.1136/ annrheumdis-2015-207492)

For numbered affiliations see end of article.

\section{Correspondence to}

Professor Bhaskar Dasgupta, Department of Rheumatology, Southend University Hospital, Prittlewell Chase, Westcliff-onSea, Essex SSO ORY, UK; Bhaskar.dasgupta@southend. nhs.uk

ELM and BD are co-senior authors.

This article is published simultaneously in the October 2015 issue of Arthritis \& Rheumatology.

Supported by the American College of Rheumatology and the European League Against Rheumatism.

Received 20 February 2015 Revised 21 May 2015 Accepted 13 July 2015

CrossMark

\section{S Linked}

- http://dx.doi.org/10.1136/ annrheumdis-2015-207578

To cite: Dejaco $C$, Singh YP, Perel $P$, et al. Ann Rheum Dis 2015;74:1799-1807.

\section{ABSTRACT}

Therapy for polymyalgia rheumatica (PMR) varies widely in clinical practice as international recommendations for PMR treatment are not currently available. In this paper, we report the 2015 European League Against Rheumatism (EULAR)/American College of Rheumatology (ACR) recommendations for the management of PMR.

We used the Grading of Recommendations, Assessment, Development and Evaluation (GRADE) methodology as a framework for the project. Accordingly, the direction and strength of the recommendations are based on the quality of evidence, the balance between desirable and undesirable effects, patients' and clinicians' values and preferences, and resource use. Eight overarching principles and nine specific recommendations were developed covering several aspects of PMR, including basic and follow-up investigations of patients under treatment, risk factor assessment, medical access for patients and specialist referral, treatment strategies such as initial glucocorticoid (GC) doses and subsequent tapering regimens, use of intramuscular GCs and disease modifying anti-rheumatic drugs (DMARDs), as well as the roles of non-steroidal anti-rheumatic drugs and nonpharmacological interventions. These recommendations will inform primary, secondary and tertiary care physicians about an international consensus on the management of PMR. These recommendations should serve to inform clinicians about best practices in the care of patients with PMR.

\section{INTRODUCTION}

There are wide variations in the treatment of polymyalgia rheumatica (PMR) with respect to glucocorticoid (GC) dosages, tapering strategies, use of disease modifying anti-rheumatic drugs (DMARDs) and duration of treatment. Up to $29-45 \%$ of patients with PMR do not adequately respond to GCs within
Guidelines and recommendations developed and/or endorsed by the American College of Rheumatology (ACR) are intended to provide guidance for particular patterns of practice and not to dictate the care of a particular patient. The ACR considers adherence to these guidelines and recommendations to be voluntary, with the ultimate determination regarding their application to be made by the physician in light of each patient's individual circumstances. Guidelines and recommendations are intended to promote beneficial or desirable outcomes but cannot guarantee any specific outcome. Guidelines and recommendations developed or endorsed by the ACR are subject to periodic revision as warranted by the evolution of medical knowledge, technology, and practice.

The American College of Rheumatology is an independent, professional, medical and scientific society which does not guarantee, warrant, or endorse any commercial product or service.

3-4 weeks. Relapses and long-term GC dependency are common. ${ }^{1-4}$ GC side effects are frequently observed, occurring in around $50 \%$ of patients, and present a further challenge. ${ }^{5}$ Well considered, international recommendations can serve to standardise practice and improve patient care.

\section{Primary objective of the recommendations}

These recommendations are intended for the management of patients with PMR in various settings and are based on clinical evidence and expert opinion including informed patient decision-making.

\section{Target population}

The target population are patients with PMR based on clinician diagnosis which may be supported by 
currently available diagnostic or classification criteria. ${ }^{3} \quad 4 \quad 7-11$ Management of PMR with concomitant giant cell arteritis (GCA), rheumatoid arthritis (RA) or other conditions that present with PMR features or mimic PMR is not addressed by these recommendations.

\section{Target users}

The target users of these recommendations are primary, secondary and tertiary care physicians (that is, general practitioners (GPs), specialists in general (internal) medicine and rheumatologists).

\section{METHODS}

For a detailed description of methods, see online supplementary file $\mathrm{S} 1$.

In brief, we used the Grading of Recommendations, Assessment, Development and Evaluation (GRADE) methodology as a framework. ${ }^{12-15}$ We formulated 12 PICO (problem/ population, intervention, comparison, and outcome) questions on interventions and 10 questions on prognostic factors (see online supplementary box S1A and S1B, or the accompanying paper by Dejaco et $a l^{16}$ ). The systematic literature review (SLR) was conducted by two investigators (CDe and YPS) using Ovid MEDLINE, Embase, PubMed, CINAHL, Web of Science and the Cochrane Library databases (from January 1970 until April 2014), and applying the thesauri of PMR, text words, abbreviations and truncated text words. Outcome parameters used in the SLR may be found in supplementary box S2. Quality appraisal of interventional and prognostic studies was performed using GRADE $^{17} 18$ and the Quality in Prognostic Studies (QUIPS) tool, ${ }^{19}$ respectively. According to GRADE methodology, a guideline panel should consider the following aspects when formulating recommendations: (1) overall quality of evidence; (2) balance between desirable and undesirable effects; (3) patients' and clinicians' values and preferences; and (4) resource use. External evidence (from other American College of Rheumatology (ACR)/ European League Against Rheumatism (EULAR) recommendations; see online supplementary table S1 for details) on safety aspects related to the use of non-steroidal anti-inflammatory drugs (NSAIDs), GCs and methotrexate (MTX) was taken into account as indirect evidence, in order to identify the optimal trade-off between the benefit and harm of interventions. Prognostic factors were used to build subgroups and to adapt the recommendations based on the presence or absence of unfavourable prognostic factors. Final recommendations were either 'in favour' or 'against' an intervention, and were graded as 'conditional' or 'strong'. A strong recommendation in favour (against) was considered when the panel was certain that benefits did (did not) outweigh risks and burdens, the preferences/values of patients were met (not met) and resource use was reasonable (unreasonably high). If uncertainty existed, a conditional recommendation was made.

\section{RESULTS}

The results of the SLR are reported in a separate manuscript. ${ }^{16}$ See online supplementary file S2 for a summary of the SLR and external evidence considered by the guideline panel.

\section{General aspects}

These recommendations should be understood as clinical advice and do not dictate the care of a particular patient. The EULAR and ACR consider adherence to these recommendations to be voluntary, with the physician making the ultimate decision to apply them in light of each patient's individual circumstances.

\section{Overarching principles for the management of PMR}

The group agreed upon several principles deemed to be fundamental aspects of clinical care in PMR as detailed in box 1 . These principles have not directly resulted from the SLR, but are consensus based. They are intended as a framework for the implementation of the specific treatment recommendations and are of a general 'overarching' nature, a concept adapted from earlier EULAR recommendations. ${ }^{20-22}$

\section{Specific recommendations}

See box 1 for a summary of the recommendations. A flow-chart for the management of PMR patients is depicted in figure 1.

Recommendation 1: (PICO 1) The panel strongly recommends using GCs instead of NSAIDs in patients with PMR, with the exception of possible short-term use of NSAIDs and/or analgesics in PMR patients with pain related to other conditions (eg, co-existing osteoarthritis). No specific recommendation can be made for analgesics.

Explanation: The group recommends strongly against the use of NSAIDs compared to GCs in the treatment of PMR since the relative harm of long-term NSAID use (as mainly indicated by external evidence) outweighs the possible small benefits in PMR. No specific recommendation can be made for analgesics. On a basis of consensus, the panel recognised that the shortterm use of NSAIDs and/or analgesics may be necessary in the setting of pain related to conditions other than PMR.

Recommendation 2: (PICO 2) The panel strongly recommends using the minimum effective individualised duration of GC therapy in PMR patients.

A more specific recommendation is not possible due to the lack of published evidence on this issue. On a basis of consensus and in accordance with the overarching principles, the group unanimously agreed to choose the minimum effective individualised duration and dose of GCs to balance benefit versus harm after assessing risk factors for GC-related adverse events, comorbidities, concomitant medications, relapses and prolonged therapy. Our recommended GC tapering schedule (see Recommendation 4) assumes a minimum of 12 months of treatment. A more specific statement is not possible because of the lack of PMR studies on this particular topic and because of the multiple subgroups and factors that need to be taken into account.

Recommendation 3: (PICOs 3-5) The panel conditionally recommends using the minimum effective GC dose within a range of $12.5-25 \mathrm{mg}$ prednisone equivalent daily as the initial treatment of PMR. A higher initial prednisone dose within this range may be considered in patients with a high risk of relapse and low risk of adverse events, whereas in patients with relevant comorbidities (eg, diabetes, osteoporosis, glaucoma, etc) and other risk factors for GC-related side effects, a lower dose may be preferred. The panel discourages conditionally the use of initial doses $\leq 7.5 \mathrm{mg} /$ day and strongly recommends against the use of initial doses $>30 \mathrm{mg} /$ day.

According to the SLR on prognostic factors and based on clinical experience, the group agreed upon the existence of various PMR subgroups that are characterised by different risks of relapse, prolonged GC therapy and/or GC-related adverse events as well as by various comorbidities and co-medications. As there are insufficient data to make evidence-based recommendations for all conceivable subgroups, and taking current clinical practice into account, ${ }^{28-30}$ the panel agreed upon the use of the minimum effective GC dose out of a range of 12.5$25 \mathrm{mg}$ prednisone equivalent daily balancing benefits versus 
Box 1 Summary of the 2015 European League Against Rheumatism (EULAR)/American College of Rheumatology (ACR) recommendations for the management of polymyalgia rheumatica (PMR)

\section{Target population:}

Patients with PMR based on clinician diagnosis which may be supported by currently available diagnostic or classification criteria. ${ }^{3} 4-11$ Overarching principles for the management of PMR:

A. Adoption of a safe and specific approach to ascertain the PMR case definition. The clinical evaluation should be directed towards exclusion of relevant mimicking (eg, non-inflammatory, inflammatory (such as giant cell arteritis or rheumatoid arthritis), drug-induced, endocrine, infective and neoplastic) conditions.

B. Every case of PMR should have the following assessments prior to the prescription of therapy (primary or secondary care):

- Documentation of a basic laboratory dataset. This will help to exclude mimicking conditions and establish a baseline for monitoring of therapy. This should include rheumatoid factor and/or anti-cyclic citrullinated peptide antibodies (ACPA), C-reactive protein and/or erythrocyte sedimentation rate (ESR), blood count, glucose, creatinine, liver function tests, bone profile (including calcium, alkaline phosphatase) and dipstick urinalysis. Additional investigations to consider are protein electrophoresis, thyroid stimulating hormone (TSH), creatine kinase and vitamin D.

- Depending on clinical signs and symptoms and the likelihood of the alternative diagnoses, additional more extensive serological tests such as anti-nuclear antibodies (ANA), anti-cytoplasmic neutrophil antibodies (ANCA) or tuberculosis tests may be performed to exclude mimicking conditions. Additional investigations such as chest radiographs may be considered at the discretion of the physician in order to exclude other diagnoses.

- Determination of comorbidities (particularly hypertension, diabetes, glucose intolerance, cardiovascular disease, dyslipidaemia, peptic ulcer, osteoporosis (and particularly recent fractures), presence of cataract or (risk factors for) glaucoma, presence of chronic or recurrent infections, and co-medication with non-steroidal anti-inflammatory drugs (NSAIDs) as outlined in Smolen et $a^{21}$ and Gossec et $a l_{1}^{22}$ other relevant medications and risk factors for steroid-related side effects. Female sex was associated with a higher risk of glucocorticoid (GC) side effects in low to moderate quality studies. ${ }^{23-25}$

- The role of risk factors for relapse/prolonged therapy is not clear yet. Baseline factors that were associated in low to moderate quality studies with a higher relapse rate and/or prolonged therapy in PMR studies were: female sex, ${ }_{1}^{24}{ }^{26}$ high ESR (>40 mm/1st hour $)^{26-31}$ and peripheral inflammatory arthritis. ${ }^{32} \mathrm{~A}$ number of equally low to moderate quality studies, however, failed to demonstrate an association between these factors and relapse/prolonged therapy. ${ }^{27-30} 32-44$

C. Consideration of specialist referral, particularly in case of atypical presentation (such as peripheral inflammatory arthritis, systemic symptoms, low inflammatory markers, age $<60$ years), experience of or high risk of therapy-related side effects, PMR refractory to GC therapy, and/or relapses/prolonged therapy.

D. Treatment of PMR patients should aim at the best care and must be based on a shared decision between the patient and the treating physician.

E. Patients should have an individualised PMR management plan. Patient perspective and preferences should be considered in the individualised choice of initial GC dose and subsequent tapering of GCs in PMR.

F. Patients should have access to education focusing on the impact of PMR and treatment (including comorbidities and disease predictors) and advice on individually tailored exercise programmes.

G. Every patient treated for PMR in primary or secondary care should be monitored with the following assessments: risk factors and evidence for steroid-related side effects, comorbidities, other relevant medications, evidence and risk factors for relapse/prolonged therapy. Continuous documentation of a minimal clinical and laboratory dataset should be conducted while prescribing GCs. Follow-up visits are suggested every 4-8 weeks in the first year, every 8-12 weeks in the second year, and as indicated in case of relapse or as prednisone is tapered and discontinued.

H. It is important for patients to have rapid and direct access to advice from doctors, nurses or trained allied healthcare staff to report any changes in their condition such as flares and adverse events.

Specific recommendations for the management of PMR patients:

1. The panel strongly recommends using GC instead of NSAIDs in patients with PMR, with the exception of possible short-term use of NSAIDs and/or analgesics in PMR patients with pain related to other conditions. No specific recommendation can be made for analgesics.

2. The panel strongly recommends using the minimum effective individualised duration of GC therapy in PMR patients.

3. The panel conditionally recommends using the minimum effective $\mathrm{GC}$ dose within a range of $12.5-25 \mathrm{mg}$ prednisone equivalent daily as the initial treatment of PMR. A higher initial prednisone dose within this range may be considered in patients with a high risk of relapse and low risk of adverse events, whereas in patients with relevant comorbidities (eg, diabetes, osteoporosis, glaucoma, etc) and other risk factors for GC-related side effects, a lower dose may be preferred. The panel discourages conditionally the use of initial doses $\leq 7.5 \mathrm{mg} /$ day and strongly recommends against the use of initial doses $>30 \mathrm{mg} /$ day.

4. The panel strongly recommends individualising dose tapering schedules, predicated to regular monitoring of patient disease activity, laboratory markers and adverse events.The following principles of GC dose tapering are suggested:

A. Initial tapering: Taper dose to an oral dose of $10 \mathrm{mg} /$ day prednisone equivalent within 4-8 weeks.

B. Relapse therapy: Increase oral prednisone to the pre-relapse dose and decrease it gradually (within 4-8 weeks) to the dose at which the relapse occurred.

C. Tapering once remission is achieved (following initial and relapse therapies): Taper daily oral prednisone by $1 \mathrm{mg}$ every 4 weeks (or by $1.25 \mathrm{mg}$ decrements using schedules such as $10 / 7.5 \mathrm{mg}$ alternate days, etc) until discontinuation given that remission is maintained. 
5. The panel conditionally recommends considering intramuscular (i.m.) methylprednisolone as an alternative to oral GCs. The choice between oral GCs and i.m. methylprednisolone remains at the discretion of the treating physician. In one clinical trial, a starting dose of $120 \mathrm{mg}$ methylprednisolone i.m. injection every 3 weeks was applied. ${ }^{23}$

6. The panel conditionally recommends using a single rather than divided daily doses of oral GCs for the treatment of PMR, except for special situations such as prominent night pain while tapering GCs below the low-dose range (prednisone or equivalent $<5 \mathrm{mg}$ daily).

7. The panel conditionally recommends considering early introduction of methotrexate (MTX) in addition to GCs, particularly in patients at a high risk for relapse and/or prolonged therapy as well as in cases with risk factors, comorbidities and/or concomitant medications where GC-related adverse events are more likely to occur. MTX may also be considered during follow-up of patients with a relapse, without significant response to GC or experiencing GC-related adverse events. MTX has been used at oral doses of $7.5-10 \mathrm{mg} /$ week in clinical trials. ${ }^{24-27}$

8. The panel strongly recommends against the use of TNF $\alpha$ blocking agents for treatment of PMR.

9. The panel conditionally recommends considering an individualised exercise programme for PMR patients aimed at the maintenance of muscle mass and function, and reducing risk of falls especially in older persons on long-term GCs as well as in frail patients.

10. The panel strongly recommends against the use of the Chinese herbal preparations Yanghe and Biqi capsules in PMR patients.

harms. The panel did not construct case vignettes as a possible aid for clinical practice; however, it is suggested that a higher initial prednisone dose (within the given range) may be used in patients with a high risk of relapse and low risk of adverse events, whereas in patients with relevant comorbidities (eg, diabetes, osteoporosis, glaucoma, etc) and other risk factors for GC-related side effects, a lower dose may be preferred.

The group conditionally discourages low $(\leq 7.5 \mathrm{mg} /$ day $)$ and strongly recommends against high $(>30 \mathrm{mg} /$ day prednisone equivalent) initial GC doses. For this statement the group extrapolated the data from randomised controlled trials, ${ }^{29} 31$ and took clinical experience, national PMR guidelines, ${ }^{28-30}$ as well as current ACR and/or EULAR recommendations on the use of GCs in rheumatic diseases into account. ${ }^{32-35}$ In addition, there is incontrovertible external evidence of harm from long-term large doses of GCs ${ }^{32-35}$ and lack of evidence for any benefit of a highdose regimen in PMR. It was unanimously agreed among the group that patients requiring high doses of GCs should be evaluated for alternate diagnoses and an alternate management plan.

Recommendation 4: (PICO 6) The panel strongly recommends individualising dose-tapering schedules, based on regular monitoring of patient disease activity, laboratory markers and adverse events.

The following principles of GC dose tapering are suggested:

A. Initial tapering: Taper dose to an oral dose of $10 \mathrm{mg} /$ day prednisone equivalent within 4-8 weeks

B. Relapse therapy: Increase oral prednisone to the pre-relapse dose and decrease it gradually (within 4-8 weeks) to the dose at which the relapse occurred.

C. Tapering once remission is achieved (following initial and relapse therapies): Taper daily oral prednisone by $1 \mathrm{mg}$ every 4 weeks (or by $1.25 \mathrm{mg}$ decrements using schedules such as $10 / 7.5 \mathrm{mg}$ on alternate days, etc) until discontinuation as long as remission is maintained.

In accordance with the overarching principles, the panel agreed upon a strong recommendation to individualise dose tapering and to regularly monitor PMR patients. Further, the panel proposed general principles for initial and post-relapse tapering of GCs (based on consensus and current clinical practice) but did not fix a schedule as in other guidelines. ${ }^{28-30}$ The panel agreed that equivalent objectives may be achieved by alternative tapering schedules. For example, a patient with a high initial prednisone dose (eg, $25 \mathrm{mg} /$ day) may have a fast initial taper followed by a more gradual decrease in the GC dose, whereas in a patient starting at a lower initial dose (eg, $12.5 \mathrm{mg} /$ day), the initial dose may be kept constant for longer and then eventually reduced.
The group suggested prednisone should be tapered by $1 \mathrm{mg} /$ 4 weeks or similar once remission is achieved. Again, the panel emphasised the important overall principle of gradual GC reduction without the need to prescribe a fixed schedule. The group further recognised that $1 \mathrm{mg}$ prednisone tablets are not available in all countries (making a reduction of $1 \mathrm{mg} / 4$ weeks unfeasible) and that other regimens such as alternate day reductions (eg, 10/ $7.5 \mathrm{mg}$ on alternate days, etc) are common clinical practice. ${ }^{28}$

Recommendation 5: (PICO 7) The panel conditionally recommends considering intramuscular (i.m.) methylprednisolone as an alternative to oral GCs. The choice between oral GCs and i.m. methylprednisolone remains at the discretion of the treating physician.

In one clinical trial, i.m. methylprednisolone was applied at a dose of $120 \mathrm{mg}$ every 3 weeks until week 9. At week 12, $100 \mathrm{mg}$ were used and subsequently, injections were continued at monthly intervals and the dose was reduced by $20 \mathrm{mg}$ every 12 weeks until week 48 . Thereafter, the dose was reduced by $20 \mathrm{mg}$ every 16 weeks until discontinuation. ${ }^{23} 36$

The panel did not specify a clinical phenotype where i.m. GCs would be appropriate or adequate therapy; however, the panel agreed that in clinical practice this preparation may be considered in cases where a lower cumulative GC dose is desirable, for example in female patients with difficult to control hypertension, diabetes, osteoporosis and/or glaucoma. ${ }^{37-39}$ Nonetheless, the panel acknowledged that there is a lack of convincing evidence showing significantly fewer side effects with $\mathrm{i}$. $\mathrm{m}$. methylprednisolone than with oral GC therapy.

The reasons why the panel did not endorse a strong recommendation for the use of i.m. methylprednisolone are the following: (1) the efficacy of i.m. methylprednisolone is supported by a single randomised controlled trial and confirmation of these data is still necessary; ${ }^{23} 36$ (2) this trial was neither designed nor powered as a non-inferiority trial and therefore, a difference between the efficacy of i.m. and oral GC cannot be excluded; (3) the trial failed to demonstrate a reduction in GC-related adverse events except for weight gain; (4) the longterm benefit of this preparation is unknown (particularly with respect to a possible reduction in GC side effects); and (5) i.m. methylprednisolone is not available in all countries.

Recommendation 6: (PICO 8) The panel conditionally recommends using a single rather than divided daily doses of oral GCs for the treatment of PMR, except for special situations such as prominent night pain while tapering GCs below the low-dose range (prednisone or equivalent $<5 \mathrm{mg}$ daily).

There are no studies available addressing this issue specifically in PMR. Based on clinical experience and because of the 
Patient fulfilling PMR case definition (primary or secondary care)

1. Assess comorbidities ${ }^{1}$, other relevant medications and other risk factors for steroid related side effects ${ }^{2}$

2. Assess possible risk factors for relapse/prolonged therapy ${ }^{3}$

3. Consider specialist referral (experience or risk of side-effects, relapse/prolonged therapy and/or atypical presentation)

4. Document minimal clinical and laboratory dataset

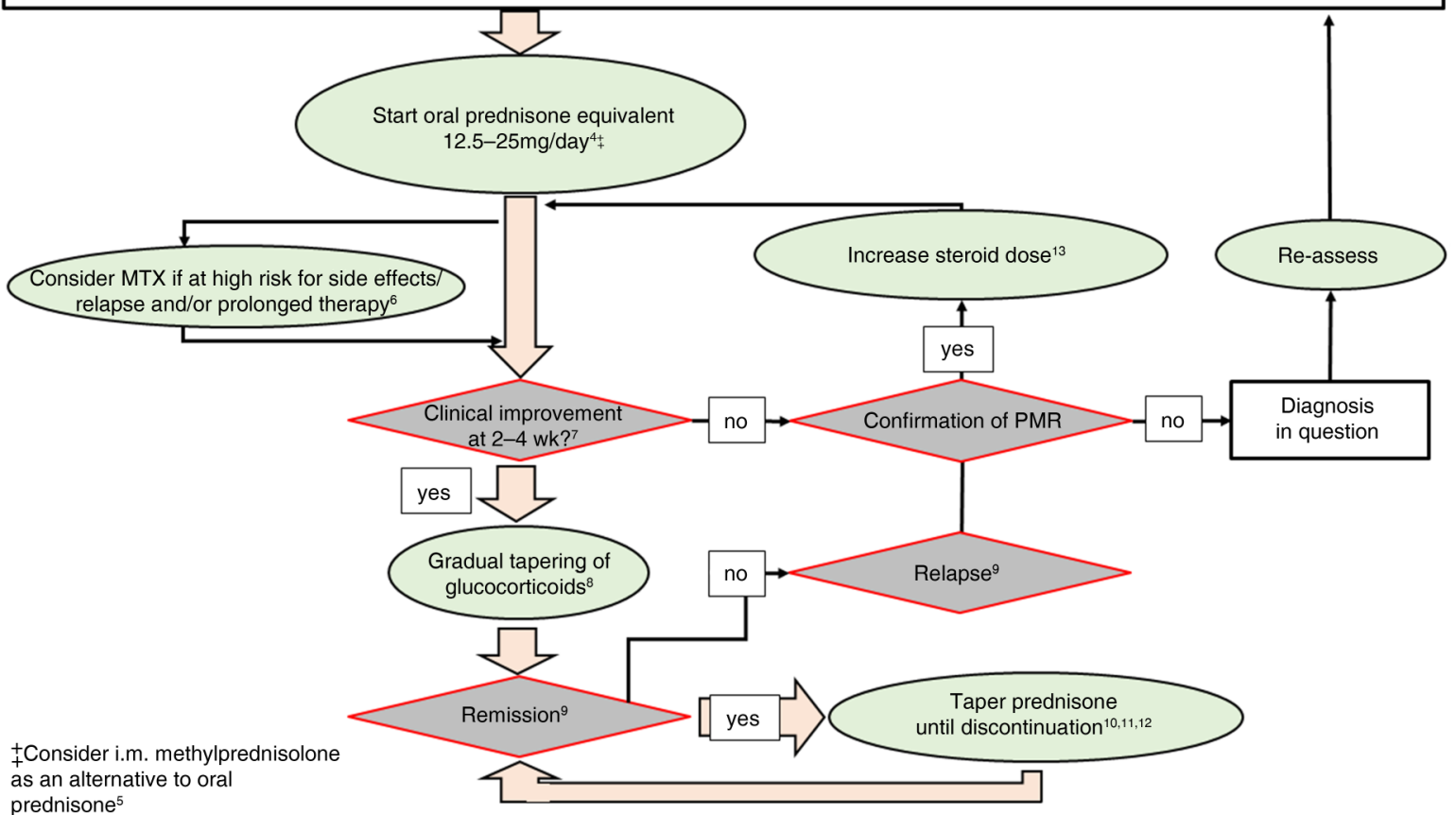

prednisone ${ }^{5}$

Figure 1 Algorithm based on the 2015 European League Against Rheumatism (EULAR)/American College of Rheumatology (ACR) recommendations for the management of polymyalgia rheumatica (PMR). The algorithm is applied to patients with PMR based on clinician

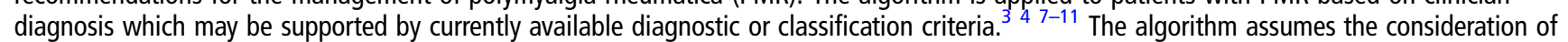
overarching principles emphasising the importance of assessing comorbidities, other relevant medications and possible risk factors for steroid-related side effects and relapse/prolonged therapy. In addition, patients diagnosed in primary care should be considered for specialist referral, particularly in case of atypical presentation (such as peripheral inflammatory arthritis, systemic symptoms, low inflammatory markers, age $<60$ years), experience or high risk of therapy-related side effects and/or relapse/prolonged therapy. A minimal clinical and laboratory dataset should be documented in each patient before prescribing therapy. ${ }^{1}$ Examples for comorbidities associated with an increased risk of glucocorticoid (GC)-related side effects are (according to Duru et $a^{32}$ and Hoes et $\left.a\right|^{35}$ ): hypertension, diabetes, glucose intolerance, cardiovascular disease, dyslipidaemia, peptic ulcer, osteoporosis (and particularly recent fractures), presence of cataract or (risk factors for) glaucoma, presence of chronic or recurrent infections, and co-medication with NSAIDs. ${ }^{2} \mathrm{~A}$ baseline factor that was associated with a higher risk of GC-related adverse events in PMR studies was: female sex. ${ }^{37-39}{ }^{3}$ The role of risk factors for relapse/prolonged therapy is not yet clear. Baseline factors that were associated with a higher relapse rate and/or prolonged therapy in PMR studies were: female sex, ${ }^{38} 47$ high erythrocyte sedimentation rate (ESR) $(>40 \mathrm{~mm} / 1 \mathrm{st} \text { hour })^{47-51} 53$ and peripheral inflammatory arthritis. ${ }^{54} \mathrm{~A}$ number of studies, however, failed to demonstrate an association between these factors and relapse. ${ }^{48-51}{ }^{54} 67-78$ Use the minimum effective dose out of a range of $12.5-25 \mathrm{mg}$ prednisone equivalent daily: a high risk of relapse/prolonged therapy favours a higher dose, while a high risk of side effects favours a lower dose. ${ }^{5} \mathrm{In}$ one randomised controlled trial, $120 \mathrm{mg}$ methylprednisolone intramuscular (i.m.) injection was used every 3 weeks as a starting dose. ${ }^{23} \mathrm{I}$.m. methylprednisolone may not be available in all countries and the possible long-term benefit in terms of efficacy and GC-sparing effects of this preparation is unknown. ${ }^{6}$ Methotrexate (MTX) has been used at oral doses of $7.5-10 \mathrm{mg} /$ week in clinical trials. ${ }^{24-27}{ }^{7} \mathrm{Clinical}$ improvement should be noted after 2 weeks, and almost complete response can be expected after 4 weeks. The definition of response criteria was beyond the scope of this project; however, a definition of response was proposed in Dasgupta et $a l^{3}{ }^{34}{ }^{8}$ For initial GC tapering, we recommend reducing the oral dose gradually to a dose of $10 \mathrm{mg} /$ day prednisone equivalent within $4-8$ weeks; after relapse therapy the dose should be decreased gradually (within 4-8 weeks) to the dose at which the relapse occurred. For i.m. methylprednisolone, a dose of $120 \mathrm{mg}$ every 3 weeks was used for the first 9 weeks in Dasgupta et al. ${ }^{23}$ No recommendation about dose adjustments of MTX can be made. ${ }^{9}$ The definition of criteria for remission and relapse was beyond the scope of this project. Definitions of remission and relapse used in clinical studies are summarised in Dejaco et al. ${ }^{65}{ }^{10}$ Once remission is achieved (following initial and relapse therapies), taper oral prednisone by $1 \mathrm{mg} / 4$ weeks (or similar, eg, $2.5 \mathrm{mg} / 10$ weeks) until discontinuation given that remission is maintained. In case i.m. methylprednisolone is used, the following tapering regimen was previously applied:23 $100 \mathrm{mg}$ methylprednisolone i.m. at week 12 , then continuation of the injections at monthly intervals with the dose reduced by $20 \mathrm{mg}$ every 12 weeks until week 48 . Thereafter, the dose was reduced by $20 \mathrm{mg}$ every 16 weeks until discontinuation. ${ }^{11}$ The group suggests that PMR patients be followed up every 4-8 weeks in the first year, every 8-12 weeks in the second year and as indicated in case of relapse or as prednisone is tapered off. ${ }^{12}$ No recommendation can be made for minimal/ optimal duration of therapy. In case patients are treated with a combination of GCs plus MTX and GCs have been withdrawn already,

discontinuation of MTX may be considered. ${ }^{13}$ Initial lack of response (eg, insufficient improvement of symptoms within 2 weeks): increase oral dose up to $25 \mathrm{mg}$ prednisone equivalent. In case i.m. methylprednisolone is used, consider switching to oral GCs. Relapse therapy: increase dose to the previously effective (ie, pre-relapse) dose.

concern that adverse events (including disturbance of the hypothalamic-pituitary-adrenal axis) may be higher with divided doses, the group agreed against the general use of divided GC doses in PMR. ${ }^{40-43}$ The effectiveness and acceptability of a single daily GC dose has been standard clinical practice in PMR and other inflammatory conditions ${ }^{44} 45$ and evening doses can cause circadian rhythm and sleep disturbances. ${ }^{46}$ 
In special situations such as in case of night pain while tapering below the low-dose range (prednisone or equivalent $<5 \mathrm{mg}$ daily), split doses may be considered. However, persistent breakthrough symptoms should prompt re-consideration of the diagnosis.

Recommendation 7: (PICO 9) The panel conditionally recommends considering early introduction of MTX in addition to GCs, particularly in patients at high risk of relapse and/or prolonged therapy as well as in cases with risk factors, comorbidities and/or concomitant medications where GC-related adverse events are more likely to occur. MTX may also be considered during follow-up in patients with a relapse, without a significant response to GC or experiencing GC-related adverse events.

In clinical trials, MTX has been used at oral doses of 7.5$10 \mathrm{mg} /$ week. ${ }^{24-27}$

Similar to the explanation of Recommendation 5, the panel felt that there is no clinical prototype unconditionally warranting treatment with MTX, rather the use of this drug should be discussed on an individual basis. In clinical practice, MTX may be considered for example in female patients ${ }^{36-39} 47$ with high initial erythrocyte sedimentation rate (ESR) $(>40 \mathrm{~mm} / 1 \mathrm{st}$ hour), ${ }^{4-53}$ peripheral inflammatory arthritis ${ }^{54}$ and/or comorbidities that may be exacerbated by GC therapy.

The panel also reached a consensus that MTX should be considered in patients who have relapsed (either on or off GCs), cases without significant response to GC or patients experiencing GC-related adverse events. The group further agreed that MTX may be used with oral or i.m. GC preparations even if the concomitant use of MTX and i.m. methylprednisolone has not been tested formally.

The efficacy of MTX was addressed in four randomised controlled trials and one retrospective study testing the use of MTX plus oral GCs (initial prednisone doses ranging from 15 to $25 \mathrm{mg} /$ day). ${ }^{24-27} 51$ There was moderate to high quality of evidence (QoE) from studies indicating a benefit of MTX regarding remission (1 study), ${ }^{27}$ relapse rate (1 study), ${ }^{24}$ discontinuation of GC (1 study) ${ }^{24}$ and cumulative GC doses (3 studies). ${ }^{24} 2627$ Evidence from one to four studies (1 related to remission, 4 to relapse, 1 to discontinuation of GC) indicating no benefit regarding these outcomes was of very low quality. ${ }^{25-27} 51$

The reasons why the panel did not support a stronger recommendation for the use of MTX in PMR are the following: (1) the total number of patients investigated in randomised trials was small $(n=194),{ }^{24-27}$ hence further confirmation of the present data is necessary; (2) results were contradictory in part, although trials with a negative result had a very low QoE; (3) a reduction in GC-related adverse events with the use of MTX has not been demonstrated. The power of the prospective studies to address this outcome, however, was insufficient. The panel nevertheless felt that earlier discontinuation of $\mathrm{GC}^{24}$ and a lower cumulative GC dose in MTX users ${ }^{26} 27$ decreases the likelihood of GC-related side effects; and (4) the cost-effectiveness of MTX use in PMR is not clear. More frequent prescriptions of MTX may lead to higher utilisation of healthcare resources in the short term (eg, because of specialist referral, monitoring visits, blood tests, etc) but may in the long term save costs by reducing GC-related side effects. Future studies are necessary to clarify this issue.

The group recognised that no recommendation can be made for the use of other non-biologic (ie, conventional synthetic and conventional targeted) DMARDs in PMR because of the lack of good evidence from PMR studies. Hydroxychloroquine was investigated by a single very low QoE retrospective study reporting no benefit regarding relapse rate. ${ }^{51}$
Recommendation 8: (PICOs 10-11) The panel strongly recommends against the use of TNF $\alpha$ blocking agents for the treatment of PMR.

The group agreed strongly against the use of TNF $\alpha$ blocking agents in PMR at this time since there is no evidence for benefit, but there is a considerable risk of potential harm and high resource use. ${ }^{55}$

No recommendation can be made for other biologic agents as no prospective trials have been published so far. There is one ongoing randomised study on the use of tocilizumab (clinicaltrials.gov NCT01396317) and another three-arm trial comparing secukinumab, canakinumab and GCs (clinicaltrials.gov NCT01364389) in PMR. The results of these studies may lead to a modification of this recommendation.

Recommendation 9: (PICO 12) The panel conditionally recommends considering an individualised exercise programme for PMR patients aimed at the maintenance of muscle mass and function, and reducing risk of falls.

There are no studies investigating the value of nonpharmacological therapies (eg, physiotherapy, relaxation techniques, diets, etc) in PMR and there is insufficient clinical experience on this issue to agree on a specific recommendation. Nevertheless, the panel agreed on recommending an individualised exercise programme (see overarching principles) in view of its benefit for maintaining muscle mass and function and reducing risk of falls, especially in older persons on long-term GCs as well as in frail patients.

\section{Use of herbal preparations in PMR}

The panel strongly recommends against the use of the Chinese herbal preparations Yanghe and Biqi capsules in PMR patients.

There were some discussions about whether herbal preparations could be considered non-pharmacological interventions (and were therefore within the scope of PICO 12); however, the panel felt the need for comment on this issue because several preparations are available which may be popular with PMR patients.

The SLR identified two studies testing Chinese Yanghe herb decoction and Chinese Biqi capsules in PMR patients. ${ }^{56} 57$ For Chinese Yanghe there is moderate QoE for a lower ESR at week 8 (mean difference $6.0 \mathrm{~mm} / \mathrm{h}$ ) and $12(6.4 \mathrm{~mm} / \mathrm{h})$ and very low QoE indicating a lower rate of GC-related adverse events (with borderline significance of the effect estimate) as well as reduced morning stiffness at week $12 .{ }^{56}$ For Biqi capsules there is low QoE indicating a higher response rate at week $12 .{ }^{57}$

The group nevertheless agreed (after balancing evidence, benefit/harm, availability and resource use) to recommend strongly against the regular use of these preparations at this time for the following reasons: (1) the relevance of the small effect of Chinese Yanghe herb decoction on ESR is minimal for patients and good evidence for a clinical benefit of the substance is not available; (2) neither of the two substances is approved by the US Food and Drug Administration (FDA) or the European Medicines Agency (EMA); (3) the generalisability of the evidence for benefit is unclear; (4) these preparations may have unforeseeable adverse effects; (5) the availability of high quality preparations is unclear; and (6) resource impact (ie, costs) is unknown.

\section{Release and implementation of the recommendations} See online supplementary file S2.

\section{Cost implications and conflicts of interest}

Cost implications are outside the scope of these recommendations.

None of the panel members disclosed any conflict of interest associated with the development of these recommendations. 


\section{Box 2 Research agenda}

The group agreed that future studies in polymyalgia rheumatica (PMR) should be multicentre and properly powered using an agreed, validated core outcome set and a robust trial design that would maximise the power of studies, facilitate regulatory approvals and allow future meta-analysis.

Specific research questions:

1. Which outcome measures including patient-related outcomes, and response, remission and relapse criteria should be used in PMR? What is the value of a composite score? What are the most relevant treatment targets in PMR?

2. What is the efficacy and safety of different routes of glucocorticoid (GC) administration (oral, intramuscular, intra-articular), different initial GC doses, various GC tapering regimens, and different GC flare doses?

3. What is the efficacy and safety of DMARDs (non-TNF $\alpha$ biologic, conventional synthetic and conventional targeted) in PMR? What is the optimal strategy for using DMARDs in PMR: monotherapy versus combination therapy, early versus late introduction, and (particularly for biologics) use with or without GCs?

4. What is the minimal/optimal duration of therapy and which strategies for withdrawing GCs and/or DMARDs yield the best efficacy/ safety profile?

5. What is the optimal strategy for shared primary and specialty care including recommendations for specialist referral? How can patients be better involved in treatment decisions, and are there any decision aids? What is the role of self-management?

6. What is the value of tight control (ie, treat to target) versus conventional management strategies in PMR?

7. How should patients with long-standing disease and long-term low-dose GC therapy be managed?

8. What is the cost utility and effectiveness of DMARD use in PMR (versus GC use alone)?

9. What is the value of non-pharmacological therapies in PMR? Particularly, it is assumed but not yet demonstrated that physiotherapy may support preservation of function and reduce the risk of adverse events related to GC use. Patients may benefit from exercise by maintaining muscle mass and function as well as by fall prevention especially in the frail. What is the role of diet in PMR and nutrition supplements (eg, fish oil) related to outcomes?

10. What is the efficacy and safety of herbal preparations in PMR?

11. What is the role of imaging (particularly ultrasound) for the assessment and monitoring of PMR, identification of overlap with other diseases (eg, large vessel vasculitis or inflammatory arthritis) alongside clinical and patient reported outcomes?

12. Which biomarkers may be useful in PMR? Why do some patients do better than others? How can we identify these groups and what is the biological mechanism behind it? Should different drugs be applied to different PMR subgroups?

13. What is the morbidity and mortality of PMR patients (with a particular focus on cardiovascular risk) in long-term observational studies?

14. What is the aetiopathogenesis of PMR? Which targeted therapies could be developed based on new knowledge of disease mechanisms?

Bolded points indicate the top 5 items of the research agenda according to the opinion of the guideline panel.

\section{DISCUSSION}

See online supplementary file S3 for a full-length discussion. The 2015 EULAR/ACR recommendations for the management of PMR is the first collaborative project between EULAR and ACR to endorse treatment recommendations in rheumatology.

We recognise that our recommendations are only partially supported by evidence, and that they do not cover all aspects important for the management of PMR. The group therefore unanimously agreed that the research agenda (covering the evidence gaps related to PMR management) is an important result of this project (box 2).

Due to our rigorous SLR approach to select high quality papers, we did not include other reviews, case reports or case series indicating possible treatment options in treatment-resistant PMR patients. For example, we found one earlier SLR reporting similar conclusions regarding the value of MTX in PMR. ${ }^{58}$ In addition, two case series were recently published on the use of leflunomide ${ }^{59} 60$ and a few case reports are available on tocilizumab. ${ }^{61-63}$ Azathioprine has been tested in a double-blind randomised controlled trial in patients with PMR and GCA; however, as PMR patients were not analysed separately, we did not include this study in the SLR. ${ }^{64}$

It was beyond the scope of this recommendation project to define treatment targets in PMR. 'Clinical improvement' was considered as the first treatment goal after the initiation of GCs, and the response criteria used in the 2012 classification criteria study may be considered. ${ }^{3}$ Remission and relapse have been heterogeneously defined in the literature, as we pointed out previously. ${ }^{65}$ Future prospective studies aimed at the validation of new definitions of response, remission and relapse are, therefore, required to enable a targeted treatment approach in PMR. ${ }^{66}$

The most important limitations of this project are the paucity of high quality trials (as mentioned above) and the fact that GRADE is less well developed for the assessment of rare outcomes. Consequently, the QoE for adverse events is usually lower than for efficacy data. This necessitated the use of relevant external evidence to strengthen this aspect of our recommendations.

These recommendations should support clinicians to achieve the best patient outcomes. Further research on existing drugs is necessary to offer additional, evidence-based treatment options to our patients. We anticipate an update of these recommendations 3 years after their publication; however, an earlier revision may be necessary if new data emerge that would modify the current recommendations.

\section{Author affiliations}

${ }^{1}$ Department of Rheumatology, Medical University Graz, Graz, Austria

${ }^{2}$ Department of Rheumatology, Southend University Hospital, Southend, UK

${ }^{3}$ Faculty of Epidemiology and Population Health, London School of Hygiene \&

Tropical Medicine, London, UK 
${ }^{4}$ Department of Health Services Research and Policy, London School of Hygiene \& Tropical Medicine, London, UK

${ }^{5}$ Department of Internal Medicine, Research Laboratory and Academic Division of Clinical Rheumatology, University of Genova, Genoa, Italy

${ }^{6}$ University of Leeds, NIHR-Leeds Musculoskeletal Biomedical Research Unit and Leeds Institute of Rheumatic and Musculoskeletal Medicine, Leeds, UK

${ }^{7}$ Department of Rheumatology, Mayo Clinic, Jacksonville, Florida, USA

${ }^{8}$ Department of Internal Medicine and Rheumatology, Military Institute of Medicine, Warsaw, Poland

${ }^{9}$ 3rd Rheumatology Department, National Institute of Rheumatology and

Physiotherapy, Budapest, Hungary

${ }^{10}$ Hoyland House General Practice, Painswick, UK

${ }^{11}$ General Practice, Bibbiano, Italy

${ }^{12}$ Department of Rheumatology, Charitè University Medicine, Berlin, Germany

${ }^{13}$ Division of Rheumatology, Allergy and Immunology, Winthrop-University Hospital

Campus, Stony Brook University School of Medicine, Mineola, New York, USA

${ }^{14}$ Department of Rheumatology, Timaru Hospital, Timaru, New Zealand

${ }^{15}$ Department of Systemic Autoimmune Diseases, University of Barcelona, Hospital Clinic, Barcelona, Spain

${ }^{16}$ Department of Rheumatology, Hospital of Southern Norway Trust, Kristiansand, Norway

${ }^{17}$ Division of Rheumatology, Brigham and Women's Hospital, Boston,

Massachusetts, USA

${ }^{18}$ Department of Internal Medicine-Clinic VI, Medical University of Innsbruck, Innsbruck, Austria

${ }^{19}$ Patient Representative from PMRGCAuk, UK

${ }^{20}$ Department of Medicine, University of Cambridge, Cambridge, UK

${ }^{21}$ Department of Rheumatology, Hospital Universitário Pedro Ernesto, Rio de Janeiro, Brazil

${ }^{22}$ Department of Internal Medicine, University of Kwa Zulu Natal, Prince Mshiyeni

Memorial Hospital, Nelson R Mandela School of Medicine, Durban, South Africa

${ }^{23}$ Arthritis Research UK Primary Care Centre, Keele University, Keele, UK

${ }^{24}$ Facultad de Medicina, Servicio de Reumatología, Universidad de Cantabria, Hospital Universitario Marqués de Valdecilla, Santander, Spain

${ }^{25}$ Department of Medicine, Division of Allergy, Clinical Immunology, and

Rheumatology, The University of Kansas Medical Center Kansas City, Kansas, USA

${ }^{26}$ Department of Family Medicine, Mayo Clinic, Rochester, Minnesota, USA

${ }^{27}$ Department of Rheumatology, NHO Kumamoto Saishunsou National Hospital,

Clinical Research Center for Rheumatic Diseases, Kohshi, Kumamoto, Japan

${ }^{28}$ Department of Rheumatology, Sahlgren University Hospital, Göteborg, Sweden

${ }^{29}$ Department of Internal Medicine, Division of Rheumatology, Azienda Ospedaliera-

IRCCS di Reggio Emilia, Reggio Emilia, Italy

${ }^{30}$ Immanuel Krankenhaus Berlin: Medical Center for Rheumatology Berlin-Buch Berlin, Berlin, Germany

${ }^{31}$ Department of Medicine, Hospital for Special Surgery, New York, New York, USA

${ }^{32}$ Patient Representative from the USA

${ }^{33}$ Department of Internal Medicine, Universidade Federal de São Paulo, São Paulo, Brazil

${ }^{34}$ Department of Health Sciences Research, Division of Rheumatology, Mayo Clinic College of Medicine, Rochester, Minnesota, USA

Twitter Follow Sarah Mackie at @Sarah_L_Mackie and Andreas P Diamantopoulos at @adiamanteas

Acknowledgements We thank Catherine Wardle from Southend library for her assistance retrieving full text articles.

Contributors All authors contributed to conception and design of the study, acquisition and/or interpretation of data, drafting the article or revising it critically for important intellectual content, and approved the final version of the manuscript. All authors had full access to all of the data (including statistical reports and tables) in the study and can take responsibility for the integrity of the data and the accuracy of the data analysis.

Funding This study was supported by research grants from the American College of Rheumatology and the European League Against Rheumatism.

Competing interests All the participants of this project made a disclosure of possible conflicts of interest. The individual declarations are available on demand at the American College of Rheumatology secretariat and are summarised below as remuneration for consultation and/or speaking engagements (' $R$ ') or industry-related research funding ('F'). AB-R: MEDAC, Abbott, ENEL-MED, Wyeth, Lilly; F: Wyeth, Lilly; PB—R: Pfizer, Csont Dg (Military Medical Institute Warsaw), Sonosite, APLAR, Abbott; F: Abbott, BMS; FB-R: Merck Serono, Horizon Pharma (formerly Nitec Pharma), Mundipharma International Ltd; F: Merck Serono, Horizon Pharma; SC-R: Centocor; MCid-R: Centocor, Roche, BMS; F: Marato TV3 (private foundation); MCim—R: Roche, BMS, Menarini; F: Roche; BD—R: Merck; F: Roche, Mundipharma, Servier; CDe—R: BMS, Pfizer, AbbVie, MSD, Merck Serono, Roche; F: Pfizer, MSD; CDu—R: BMS, Pfizer, AbbVie, MSD, Merck Serono, Roche; DJ—R, F: Roche/ Genentech; SMa—R: Pfizer, Napp Pharmaceuticals, AstraZeneca; AM—R:
AstraZeneka, Pfizer; VM-T—R: UCB-Pharma, Pfizer, UCB Pharma, Cellerix, Abbott; F: Roche; ELM-F: Ardea, Sanofi, Centocor-Jansen, Celgene, Amgen, Roche,

Genentech, Mesoblast, Novartis, Pfizer, UCB Pharma; SMo—F: Bristol-Myers Squibb; CS—R: Novartis; MS—R: Abbott, Pfizer, Amgen, GSK, MSD; WS—R: Berlin-Chemie, Medac, Pfizer, AbbVie, Roche, Mundipharma, UCB, MSD; F: Mundipharma, Novartis, MJD, Actelion, GE, Esadie, Savient; RS—F: funded research by Roche-Genetech.

Provenance and peer review Not commissioned; externally peer reviewed.

\section{REFERENCES}

1 Hutchings A, Hollywood J, Lamping DL, et al. Clinical outcomes, quality of life, and diagnostic uncertainty in the first year of polymyalgia rheumatica. Arthritis Rheum 2007:57:803-9.

2 Dejaco C, Duftner C, Dasgupta B, et al. Polymyalgia rheumatica and giant cell arteritis: management of two diseases of the elderly. Aging Health 2011;7:633-45.

3 Dasgupta B, Cimmino MA, Maradit Kremers H, et al. 2012 Provisional classification criteria for polymyalgia rheumatica: a European League Against Rheumatism/ American College of Rheumatology collaborative initiative. Arthritis Rheum 2012;64:943-54.

4 Dasgupta B, Cimmino MA, Maradit-Kremers H, et al. 2012 Provisional classification criteria for polymyalgia rheumatica: a European League Against Rheumatism/ American College of Rheumatology collaborative initiative. Ann Rheum Dis 2012;71:484-92.

5 Curtis JR, Westfall AO, Allison J, et al. Population-based assessment of adverse events associated with long-term glucocorticoid use. Arthritis Rheum 2006;55:420-6.

6 McDonough AK, Curtis JR, Saag KG. The epidemiology of glucocorticoid-associated adverse events. Curr Opin Rheumatol 2008;20:131-7.

7 Chuang TY, Hunder GG, Ilstrup DM, et al. Polymyalgia rheumatica: a 10-year epidemiologic and clinical study. Ann Intern Med 1982:97:672-80.

8 Healey LA. Long-term follow-up of polymyalgia rheumatica: evidence for synovitis. Semin Arthritis Rheum 1984;13:322-8.

9 Bird HA, Esselinckx W, Dixon AS, et al. An evaluation of criteria for polymyalgia rheumatica. Ann Rheum Dis 1979:38:434-9.

10 Jones JG, Hazleman BL. Prognosis and management of polymyalgia rheumatica. Ann Rheum Dis 1981:40:1-5.

11 Hamrin B. Polymyalgia arteritica. Acta Med Scand Supp/ 1972;533:1-131.

12 Guyatt GH, Oxman AD, Schünemann HJ, et al. GRADE guidelines: a new series of articles in the Journal of Clinical Epidemiology. I Clin Epidemiol 2011;64:380-2.

13 Brożek JL, AKI EA, Compalati E, et al. Grading quality of evidence and strength of recommendations in clinical practice guidelines part 3 of 3 . The GRADE approach to developing recommendations. Allergy 2011;66:588-95.

14 Brozek JL, AkI EA, Jaeschke R, et al. Grading quality of evidence and strength of recommendations in clinical practice guidelines: Part 2 of 3. The GRADE approach to grading quality of evidence about diagnostic tests and strategies. Allergy 2009;64:1109-16.

15 Brożek JL, Akl EA, Alonso-Coello P, et al. Grading quality of evidence and strength of recommendations in clinical practice guidelines. Allergy 2009;64:669-77.

16 Dejaco C, Singh Y, Perel P, et al. Current evidence for therapeutic interventions and prognostic factors in Polymyalgia Rheumatica: a Systematic Literature Review informing the 2015 European League Against Rheumatism/American College of Rheumatology recommendations for the management of Polymyalgia Rheumatica. Ann Rheum Dis 2015;74:1808-17.

17 Balshem H, Helfand M, Schünemann HJ, et al. GRADE quidelines: 3. Rating the quality of evidence. J Clin Epidemiol 2011;64:401-6.

18 Grade Working Group. GRADE homepage. http://www.gradeworkinggroup.org/ index.htm

19 Hayden JA, Côté P, Bombardier C. Evaluation of the quality of prognosis studies in systematic reviews. Ann Intern Med 2006;144:427-37.

20 Smolen JS, Landewe R, Breedveld FC, et al. EULAR recommendations for the management of rheumatoid arthritis with synthetic and biological disease-modifying antirheumatic drugs. Ann Rheum Dis 2010;69:964-75 [published erratum appears in Ann Rheum Dis 2011;70:519].

21 Smolen JS, Landewé R, Breedveld FC, et al. EULAR recommendations for the management of rheumatoid arthritis with synthetic and biological disease-modifying antirheumatic drugs: 2013 update. Ann Rheum Dis 2014;73:492-509.

22 Gossec L, Smolen IS, Gaujoux-Viala C, et al. European League Against Rheumatism recommendations for the management of psoriatic arthritis with pharmacological therapies. Ann Rheum Dis 2012;71:4-12.

23 Dasgupta B, Dolan AL, Panayi GS, et al. An initially double-blind controlled 96 week trial of depot methylprednisolone against oral prednisolone in the treatment of polymyalgia rheumatica. Br I Rheumatol 1998;37:189-95.

24 Caporali R, Cimmino MA, Ferraccioli G, et al. Prednisone plus methotrexate for polymyalgia rheumatica: a randomized, double-blind, placebo-controlled trial. Ann Intern Med 2004;141:493-500.

25 Van der Veen MJ, Dinant HJ, van Booma-Frankfort C, et al. Can methotrexate be used as a steroid sparing agent in the treatment of polymyalgia rheumatica and giant cell arteritis? Ann Rheum Dis 1996;55:218-23 [published erratum appears in Ann Rheum Dis 1996;55:563]. 
26 Ferraccioli G, Salaffi F, De Vita S, et al. Methotrexate in polymyalgia rheumatica: preliminary results of an open, randomized study. J Rheumatol 1996;23:624-8.

27 Nazarinia AM, Moghimi J, Toussi J. Efficacy of methotrexate in patients with polymyalgia rheumatica. Koomesh 2012;14:265-70.

28 Dasgupta B, Borg FA, Hassan N, et al. BSR and BHPR guidelines for the management of polymyalgia rheumatica. Rheumatology (Oxford) 2010;49: 186-90.

29 NHG. NHG-Standaard Polymyalgia rheumatica en arteriitis temporalis. https://www. nhg.org/standaarden/volledig/nhg-standaard-polymyalgia-rheumatica-en-arteriitistemporalis (accessed 8 Apr 2014).

30 Schmidt WA, Gromnica Ihle E. Polymyalgia rheumatica und Riesenzellarteriitis (Arteriitis temporalis). In: Busse 0, Fleig W, Mayet W, et al., eds. Rationelle Diagnostik und Therapie in der Inneren Medizin: Leitlinien-basierte Empfehlungen für die Praxis. Elsevier, 2011

31 Kyle V, Hazleman BL. Treatment of polymyalgia rheumatica and giant cell arteritis. I. Steroid regimens in the first two months. Ann Rheum Dis 1989:48:658-61.

32 Duru N, van der Goes MC, Jacobs JWG, et al. EULAR evidence-based and consensus-based recommendations on the management of medium to high-dose glucocorticoid therapy in rheumatic diseases. Ann Rheum Dis 2013;72:1905-13.

33 Grossman JM, Gordon R, Ranganath VK, et al. American College of Rheumatology 2010 recommendations for the prevention and treatment of glucocorticoid-induced osteoporosis. Arthritis Care Res (Hoboken) 2010;62:1515-26 [published erratum appears in Arthritis Care Res (Hoboken) 2012;64:464].

34 Van der Goes MC, Jacobs JWG, Boers M, et al. Monitoring adverse events of lowdose glucocorticoid therapy: EULAR recommendations for clinical trials and daily practice. Ann Rheum Dis 2010;69:1913-19.

35 Hoes JN, Jacobs JWG, Boers $M$, et al. EULAR evidence-based recommendations on the management of systemic glucocorticoid therapy in rheumatic diseases. Ann Rheum Dis 2007:66:1560-7.

36 Dolan AL, Moniz C, Dasgupta B, et al. Effects of inflammation and treatment on bone turnover and bone mass in polymyalgia rheumatica. Arthritis Rheum 1997:40:2022-9.

37 Cimmino MA, Moggiana G, Montecucco C, et al. Long term treatment of polymyalgia rheumatica with deflazacort. Ann Rheum Dis 1994;53:331-3.

38 Cimmino MA, Parodi M, Caporali R, et al. Is the course of steroid-treated polymyalgia rheumatica more severe in women? Ann N Y Acad Sci 2006;1069:315-21.

39 Ayoub WT, Franklin CM, Torretti D. Polymyalgia rheumatica. Duration of therapy and long-term outcome. Am J Med 1985;79:309-15.

40 Grant SD, Forsham PH, DiRaimondo VC. Suppression of 17-hydroxycorticosteroids in plasma and urine by single and divided doses of triamcinolone. $N$ Engl J Med 1965:273:1115-18.

41 Haus E, Sackett-Lundeen L, Smolensky MH. Rheumatoid arthritis: circadian rhythms in disease activity, signs and symptoms, and rationale for chronotherapy with corticosteroids and other medications. Bull NYU Hosp Jt Dis 2012;70(Suppl 1):3-10.

42 Angeli A. Circadian ACTH-adrenal rhythm in man. Chronobiologia 1974;1(Suppl 1): 253-70

43 Nichols T, Nugent CA, Tyler FH. Diurnal variation in suppression of adrenal function by glucocorticoids. J Clin Endocrinol Metab 1965;25:343-9.

44 Di Raimondo VC, Forsham PH. Some clinical implications of the spontaneous diurnal variation in adrenal cortical secretory activity. Am J Med 1956;21:321-3.

45 Diraimondo VC, Forsham PH. Pharmacophysiologic principles in the use of corticoids and adrenocorticotropin. Metabolism 1958;7:5-24.

46 DeAndrade JR. Pituitary-adrenocortical reserve during corticosteroid therapy: a report on the methopyrapone test in ten patients taking long-continued small doses. J Clin Endocrinol Metab 1964;24:261-2.

47 Barraclough K, Liddell WG, du Toit J, et al. Polymyalgia rheumatica in primary care: a cohort study of the diagnostic criteria and outcome. Fam Pract 2008:25:328-33

48 Maradit Kremers H, Reinalda MS, Crowson CS, et al. Relapse in a population based cohort of patients with polymyalgia rheumatica. J Rheumatol 2005;32: 65-73.

49 Salvarani C, Boiardi L, Mantovani V, et al. HLA-DRB1 alleles associated with polymyalgia rheumatica in northern Italy: correlation with disease severity. Ann Rheum Dis 1999;58:303-8.

50 Cantini F, Salvarani C, Olivieri I, et al. Erythrocyte sedimentation rate and C-reactive protein in the evaluation of disease activity and severity in polymyalgia rheumatica: a prospective follow-up study. Semin Arthritis Rheum 2000:30:17-24

51 Lee JH, Choi ST, Kim JS, et al. Clinical characteristics and prognostic factors for relapse in patients with polymyalgia rheumatica (PMR). Rheumatol Int 2013;33:1475-80.
52 Myklebust G, Gran JT. Prednisolone maintenance dose in relation to starting dose in the treatment of polymyalgia rheumatica and temporal arteritis. A prospective twoyear study in 273 patients. Scand I Rheumatol 2001;30:260-7.

53 Gonzalez-Gay MA, Rodriguez-Valverde V, Blanco R, et al. Polymyalgia rheumatica without significantly increased erythrocyte sedimentation rate. A more benign syndrome. Arch Intern Med 1997;157:317-20.

54 Salvarani C, Cantini F, Macchioni P, et al. Distal musculoskeletal manifestations in polymyalgia rheumatica: a prospective followup study. Arthritis Rheum 1998:41:1221-6.

55 Ramiro S, Gaujoux-Viala C, Nam JL, et al. Safety of synthetic and biological DMARDs: a systematic literature review informing the 2013 update of the EULAR recommendations for management of rheumatoid arthritis. Ann Rheum Dis 2014:73:529-35

56 Fu HZ. Clinical observation on effect of modified Yanghe Decoction combined with glycocorticoid for treatment of polymyalgia rheumatica. Zhongguo Zhong Xi Yi Jie He Za Zhi 2007;27:894-7.

57 Wang B, Zhang B, Wang H. Clinical observation about 18 cases of polymyalgia rheumatica by using Biqi capsule. China J Tradit Chinese Med Pharm 2009;24:596-8

58 Hernandez-Rodriguez J, Cid MC, Lopez-Soto A, et al. Treatment of polymyalgia rheumatica: a systematic review. Arch Intern Med 2009:169:1839-50.

59 Diamantopoulos AP, Hetland H, Myklebust G. Leflunomide as a corticosteroidsparing agent in giant cell arteritis and polymyalgia rheumatica: a case series. Biomed Res Int 2013:2013:120638.

60 Adizie T, Christidis D, Dharmapaliah C, et al. Efficacy and tolerability of leflunomide in difficult-to-treat polymyalgia rheumatica and giant cell arteritis: a case series. Int J Clin Pract 2012;66:906-9.

61 Al Rashidi A, Hegazi MO, Mohammad SA, et al. Effective control of polymyalgia rheumatica with tocilizumab. J Clin Rheumatol 2013;19:400-1.

62 Macchioni $\mathrm{P}$, Boiardi L, Catanoso M, et al. Tocilizumab for polymyalgia rheumatica: report of two cases and review of the literature. Semin Arthritis Rheum 2013:43:113-18

63 Hagihara K, Kawase I, Tanaka T, et al. Tocilizumab ameliorates clinical symptoms in polymyalgia rheumatica. J Rheumatol 2010;37:1075-6.

64 De Silva M, Hazleman BL. Azathioprine in giant cell arteritis/polymyalgia rheumatica: a double-blind study. Ann Rheum Dis 1986;45:136-8.

65 Dejaco C, Duftner C, Cimmino MA, et al. Definition of remission and relapse in polymyalgia rheumatica: data from a literature search compared with a Delphibased expert consensus. Ann Rheum Dis 2011;70:447-53.

66 Smolen JS, Aletaha D, Bijlsma JW, et al. Treating rheumatoid arthritis to target: recommendations of an international task force. Ann Rheum Dis 2010;69:631-7 [published erratum appears in Ann Rheum Dis 2011;70:1519].

67 González-Gay MA, García-Porrúa C, Vázquez-Caruncho M, et al. The spectrum of polymyalgia rheumatica in northwestern Spain: incidence and analysis of variables associated with relapse in a 10 year study. J Rheumatol 1999;26:1326-32.

68 Paulsen S. Polymyalgia rheumatica. Long term treatment with steroids. Ugesk Laeger 1971;133:944-5.

69 Salvarani C, Cantini F, Niccoli L, et al. Acute-phase reactants and the risk of relapse/recurrence in polymyalgia rheumatica: a prospective followup study. Arthritis Rheum 2005:53:33-8.

70 Caplanne D, Le Parc JM, Alexandre JA. Interleukin-6 in clinical relapses of polymyalgia rheumatica and giant cell arteritis. Ann Rheum Dis 1996:55:403-4.

71 Kanemaru K, Nagura H, Ooyama T, et al. Report of 6 cases with polymyalgia rheumatica and a review of the literature. Nihon Ronen Igakkai Zasshi 1986;23:469-76.

72 Nagaoka S, Ohno M, Ohno S, et al. Long-term outcome for patients with polymyalgia rheumatica. Rinshou Ryumachi 2000;12:348-52.

73 Larrosa M, Gratacos J, Sala M. Polymyalgia rheumatica with low erythrocyte sedimentation rate at diagnosis. J Rheumatol 2000;27:1815-16.

74 Proven A, Gabriel SE, O'Fallon WM, et al. Polymyalgia rheumatica with low erythrocyte sedimentation rate at diagnosis. J Rheumatol 1999;26:1333-7.

75 Kimura M, Tokuda Y, Oshiawa $\mathrm{H}$, et al. Clinical characteristics of patients with remitting seronegative symmetrical synovitis with pitting edema compared to patients with pure polymyalgia rheumatica. J Rheumatol 2012;39:148-53.

76 Ceccato F, Roverano SG, Papasidero S, et al. Peripheral musculoskeletal manifestations in polymyalgia rheumatica. JCR J Clin Rheumatol 2006;12:167-71.

77 Mackie SL, Hensor EM, Haugeberg G, et al. Can the prognosis of polymyalgia rheumatica be predicted at disease onset? Results from a 5-year prospective study. Rheumatology (Oxford) 2010;49:716-22.

78 Kanik KS, Bridgeford PH, Germain BF, et al. Polymyalgia rheumatica with a low erythrocyte sedimentation rate: Comparison of 10 cases with 10 cases with high erythrocyte sedimentation rate. J Clin Rheumatol 1997;3:319-23. 